\title{
The protective effect of Lonicera japonica polysaccharide on mice with depression by inhibiting NLRP3 inflammasome
}

\author{
Ping Liu ${ }^{1,2}$, Xinyu Bai ${ }^{2}$, Tao Zhang ${ }^{3}$, Limei Zhou ${ }^{2,3}$, Jing Li $^{4}$, Lan Zhang ${ }^{1}$ \\ ${ }^{1}$ Department of Pharmacy, Xuanwu Hospital of Capital Medical University, National Clinical Research Center for Geriatric Diseases, Beijing \\ Engineering Research Center for Nervous System Drugs, Beijing Institute for Brain Disorders, Key Laboratory for Neurodegenerative Diseases of \\ Ministry of Education, Beijing 100053, China; ${ }^{2}$ Key Laboratory of Basic Pharmacology of Ministry of Education and Joint International Research \\ Laboratory of Ethnomedicine of Ministry of Education, Zunyi Medical University, Zunyi 563000, China; ${ }^{3}$ Department of Laboratory Medicine, \\ Affiliated Hospital of Zunyi Medical University, Zunyi 563003, China; ${ }^{4}$ Academy of Traditional Chinese Medicine, Liaoning University of \\ Traditional Chinese Medicine, Shenyang 110032, China \\ Contributions: (I) Conception and design: P Liu; (II) Administrative support: L Zhang; (III) Provision of study material: T Zhang; (IV) Collection \\ and assembly of data: P Liu, X Bai; (V) Data analysis and interpretation: L Zhou, J Li; (VI) Manuscript writing: All authors; (VII) Final approval of \\ manuscript: All authors. \\ Correspondence to: Lan Zhang. Department of Pharmacy, Xuanwu Hospital of Capital Medical University, National Clinical Research Center for Geriatric \\ Diseases, Beijing Engineering Research Center for Nervous System Drugs, Beijing Institute for Brain Disorders, Key Laboratory for Neurodegenerative \\ Diseases of Ministry of Education, Beijing 100053, China. Email: lanizhg@126.com.
}

\begin{abstract}
Background: Depression is associated with the abnormal activation of the human inflammatory response system, which is a life-threatening disorder affecting millions of people of all ages around the world. The purposes of the present study were to examine the effect of Lonicera japonica polysaccharide (LJP), a polysaccharide extracted from Lonicera japonica Thunb., on depressed mice treated with the unpredictable emotional stress stimulation.

Methods: The components of LJP after extraction were detected by HPLC. Depression model is established through chronic unpredictable stimulation, and the depression behavior was assessed by mouse behavioral assessment, including open field, elevated plus maze, tail suspension, forced swim. Pathological changes in hippocampus of mice were observed by $\mathrm{HE}$ and toluidine blue staining. Protein expression of NLRP3 inflammasome pathway was detected by WB.

Results: It was showed that LJP mainly consisted of 8.7\% GalA, 8.2\% Rha, 16.2\% Gal, 19.5\% Ara, 26.9\% Glc and 20.5\% Man, with the average molecular weight approximately ranging from 1 to $1,000 \mathrm{kDa}$, which could significantly reduce the time in the open arms and the immobility time of the depressed mice in behavioral tests, and the expressions of NLRP3, IL-1 $\beta$, and caspase-1 in the hippocampus of depressed mice were significantly upregulated.

Conclusions: LJP exhibited a strong protective effect on mice with depression by inhibiting NLRP3 inflammasome. The results will help to understand the potential use of Lonicera japonica Thun polysaccharide in pharmaceutical and food applications better.
\end{abstract}

Keywords: Lonicera japonica; polysaccharide; depression; anti-inflammatory; NLRP3

Submitted Oct 18, 2019. Accepted for publication Nov 26, 2019.

doi: $10.21037 /$ atm.2019.12.64

View this article at: http://dx.doi.org/10.21037/atm.2019.12.64

\section{Introduction}

Depression is a serious life-threatening disorder that affects hundreds of millions of patients and may occur suddenly at all ages (1). This severe psychiatric disorder is the 4th leading cause of disability worldwide, which should be looked at more seriously, and it has a prevalence of above $15 \%$ (2). Depression is not a single disorder, but 


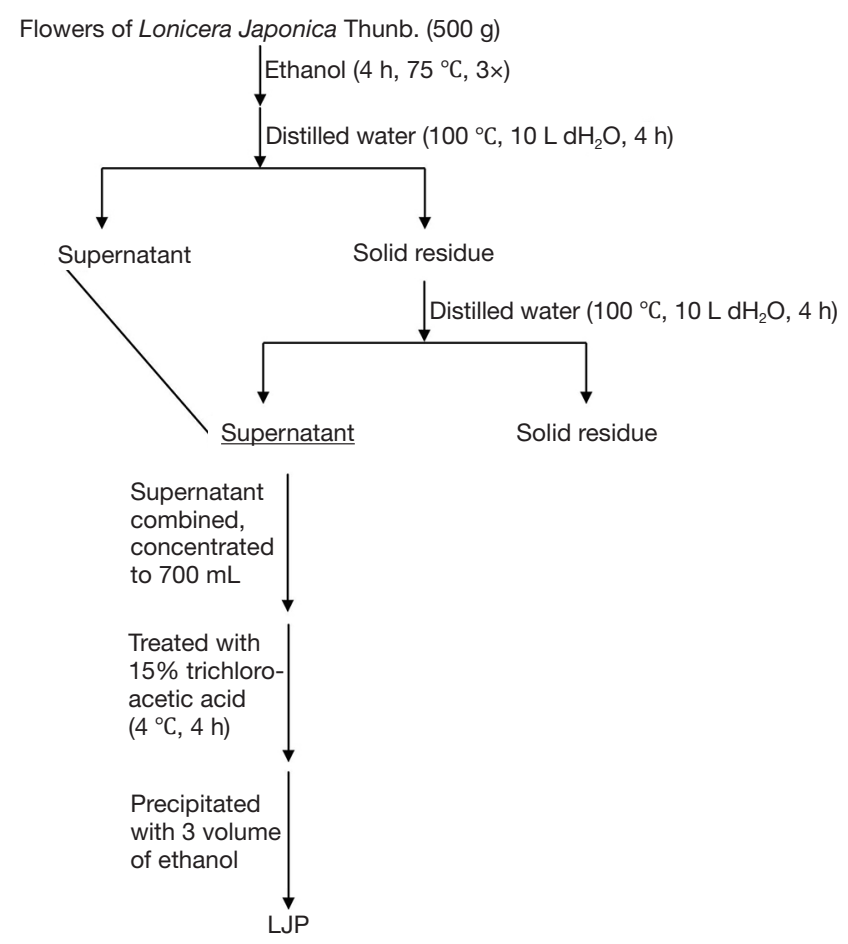

Figure 1 Extraction scheme for LJP. LJP, Lonicera japonica polysaccharide.

a heterogeneous syndrome which comprises of several symptoms of distinct causes and pathophysiology (3). According to the previous study, in many depressed patients, the ineffectiveness of conventional antidepressants causes further research on the pathogenic mechanism of depression to be required (4).

Some hypotheses are suggesting that depression is caused by the key role of neurotransmitters associated with other alterations, such as mitochondrial dysfunction and oxidative stress (5-9). Based on some evidence, depression is associated with some important cytokinesis: accompanied by activation of inflammatory pathways and reflected by an increased level of inflammatory cytokines (such as TNF- $\alpha$, IL-6, IL-1 $\beta$, etc.) $(7,9,10)$. Antidepressant effects of antiinflammatory medications and anti-inflammatory effects of antidepressants are also suggested by small-scale studies (11-14). In addition, recent studies have shown that NLRP3 (nod-like receptor protein 3) inflammasome engages in the pathological processes of psychiatric disorders, as the pathogenesis of depression (4). NLRP3 inflammasome is a multiprotein complex in cells, included procaspase-1 precursor and adaptor protein ASC (15). However, the mechanism which mediates the progression of depression of
NLRP3 inflammasome remains elusive (4).

Lonicera japonica Thunb is a traditional medicine in China, which has been listed in the "Pharmacopoeia of the People's Republic of China" database. It has been widely used to treat acute diseases, such as rheumatoid arthritis, upper respiratory tract infections, throat inflammations, infected wounds, hepatitis, dysentery, fever, and measles (16). Pharmacological studies demonstrate that Jin Yin Hua extracts and their active principles have anti-inflammatory, antibiotic, antimicrobial, antipyretic, antioxidative, antiviral, lipidlowering, hepatoprotective, and cytoprotective effects (17-21). However, there is no evidence showing that antidepressant treatment of Jin Yi Hua on depression. In the present study, Lonicera japonica polysaccharides (LJPs) were utilized to investigate its protective effect on depressed mice.

\section{Methods}

\section{Extraction of LFP}

As shown in Figure 1, the flowers of Lonicera japonica Thun, were first extracted with ethanol (95\%) under reflux for $12 \mathrm{~h} \mathrm{(} 4 \mathrm{~h} /$ time, three times) to remove hydrophobic compounds, and then the remains (about $500 \mathrm{~g}$ ) was extracted with hot distilled water $\left(10 \mathrm{~L}, 100{ }^{\circ} \mathrm{C}\right)$ two times ( $4 \mathrm{~h}$ for each). The extractions were filtered, then combined and concentrated the total volume to $700 \mathrm{~mL}$. Removal of protein impurities with $15 \%$ trichloroacetic acid $\left(4 \mathrm{~h}, 4{ }^{\circ} \mathrm{C}\right)$. After neutralization, the extract was placed in $75 \%$ absolute ethanol to obtain a precipitate which was collected and dried by vacuum to obtain LJP (yield 5.1\%, w/w).

\section{Sugar composition analysis of LFP}

LJP (2 mg) was treated with $2 \mathrm{M} \mathrm{HCl}$ in methanol for $16 \mathrm{~h}$ $\left(80{ }^{\circ} \mathrm{C}\right)$ and then with $2 \mathrm{M}$ TFA for $1 \mathrm{~h}\left(120^{\circ} \mathrm{C}\right)$. 1-phenyl3-methyl-5-pyrazolone (PMP) was used for derivatization of the monosaccharide released, and Waters e2695 HPLC analyzed the derivatives with a Dikma Platisil ODS (250 mm $\times 4.6 \mathrm{~mm})$. Waters $2489 \mathrm{UV}$ was selected as the detector. The column was eluted with $82 \%$ PBS (0.1 M, pH 7.0) and $18 \%$ acetonitrile $(\mathrm{v} / \mathrm{v})$ at a flow rate of $1 \mathrm{~mL} / \mathrm{min}$ and monitored by UV absorbance at $245 \mathrm{~nm}$ (22).

\section{The average molecular weight distribution of LFP}

The average molecular weight was determined by using gel permeation chromatography with a TSK-gel G-4000PWxl 
column (7.8 mm $\times 300 \mathrm{~mm}$, TOSOH, Japan) on a Shimadzu HPLC system with RI detector, as described previously (22). Column was pre-calibrated using dextrans $(2,000,800,500$, $100,10 \mathrm{kDa})$. The average molecular weight of LJP was calculated by linear regression analysis.

\section{Animals}

Male KM mice, which were 6-8 weeks old, were obtained from the Experimental Animal Centre of Army Medical University (Chongqing, China) and were used as experimental animals. Each mouse weighed $20 \pm 2 \mathrm{~g}$ and was maintained under specific pathogen-free conditions. The mice were reared in the animal room at a constant temperature of $25 \pm 5{ }^{\circ} \mathrm{C}$ and relative humidity of $70 \% \pm 20 \%$ with a light-dark cycle per 12 hours. The experimental protocol was approved and approved by the Ethics Committee of Zunyi Medical University.

\section{Establishment of mice model with depression and treatment method}

The unpredictable emotional stress stimulation methods were used to establish a depressed mice model in this experiment, including tail suspension experiment, natural enemy stress test, water ban, seven kinds of stress stimuli such as fasting, restraint, $24 \mathrm{~h}$ darkness/lighting, tilting. According to the principle of at least two days of non-repetition, a random stimulation is taken every day for 21 days, so that the animal is in a state of stress for a long time.

Forty KM mice were randomly numbered and divided into five groups based on random numbers, which were control group (saline, $10 \mathrm{~mL} / \mathrm{kg}$, i.g.); model group(depression); LJP (30 mg/kg, i.g.) group; LJP (100 mg/kg, i.g.) group; fluoxetine (FLU, $18 \mathrm{mg} / \mathrm{kg}$, i.g.) group, respectively. LJP and FLU (Lilly S.A.) were prepared with distilled water, which was given daily 60 minutes before stress stimulation until the end of the experiment.

\section{Behavioral tests in mice}

\section{Behavioral test of open-field}

The open-field experimental device is a square $(26 \mathrm{~cm} \times$ $26 \mathrm{~cm}$ ) placed on the ground, wherein a region centered on the center point $(13 \mathrm{~cm} \times 13 \mathrm{~cm})$ is defined as a "central region", and the rest is defined as a "peripheral region". At the beginning of the test, the corners of the peripheral region were used as a starting point, allowing the mice to explore the open field for 5 minutes freely. The camera is used to record the movement of the mice. The background color of the device is blue. The video analysis system (Noldus Systems, The Netherlands) can accurately track the video. The time at which mice enter the central and peripheral regions is recorded separately. At the end of the test, the mice were removed and placed back in the cage. Clean the experimental device and wipe with alcohol to remove the interference of the odor on the experiment.

\section{Behavioral test of elevated plus maze}

The device of the elevated cross maze consists of two vertically intersecting arms and brackets. The height of the brackets is $50 \mathrm{~cm}$, and each arm is $66 \mathrm{~cm}$. The intersection of the two arms is a square $(6 \mathrm{~cm} \times 6 \mathrm{~cm})$ area called the "central area". Both arms are $6 \mathrm{~cm}$ wide, one of which has a $20 \mathrm{~cm}$ high baffle, called the "closed wall," and the other is called the "open arm". The test began by placing the mouse in the center of the closed arm. The camera was used to record the movement of the mouse. The background color of the device was blue, and the video analysis system (Noldus Systems, The Netherlands) could accurately track the video. The time the mouse enters, the open and closed walls will be recorded. At the end of the test, the mice were removed and placed back in the cage. The experimental device was cleaned and wiped with alcohol to remove the interference of the odor on the text.

\section{Behavioral test of tail suspension}

The tails of the mice were fixed with tape on the edge of a $60 \mathrm{~cm}$ metal rod above the table, keeping the mouse head hanging downward. Note that the tape is entangled to prevent injury to the tail of the mouse. The experiment used a camera to record the movement of the mouse. The background color of the device was black. The video analysis system (Noldus Systems, Netherlands) can accurately track the video. When the mouse stops struggling and remains stationary, it is judged to be stationary. It was not recorded within $1 \mathrm{~min}$, and the immobility time was recorded after 4 minutes. At the end of the test, the mice are removed and put back in the cage.

\section{Behavioral test of forced swim}

The device of the test was a cylindrical water tank (height, $50 \mathrm{~cm}$; diameter, $15 \mathrm{~cm}$ ), $15 \mathrm{~cm}$ deep water is placed in the water tank, and the water temperature is kept at $22 \pm 2{ }^{\circ} \mathrm{C}$, the test lasted for 5 minutes. The necessary actions performed below the surface of the water do not interfere with the mouse, except that the mouse head is not sunk. The test 

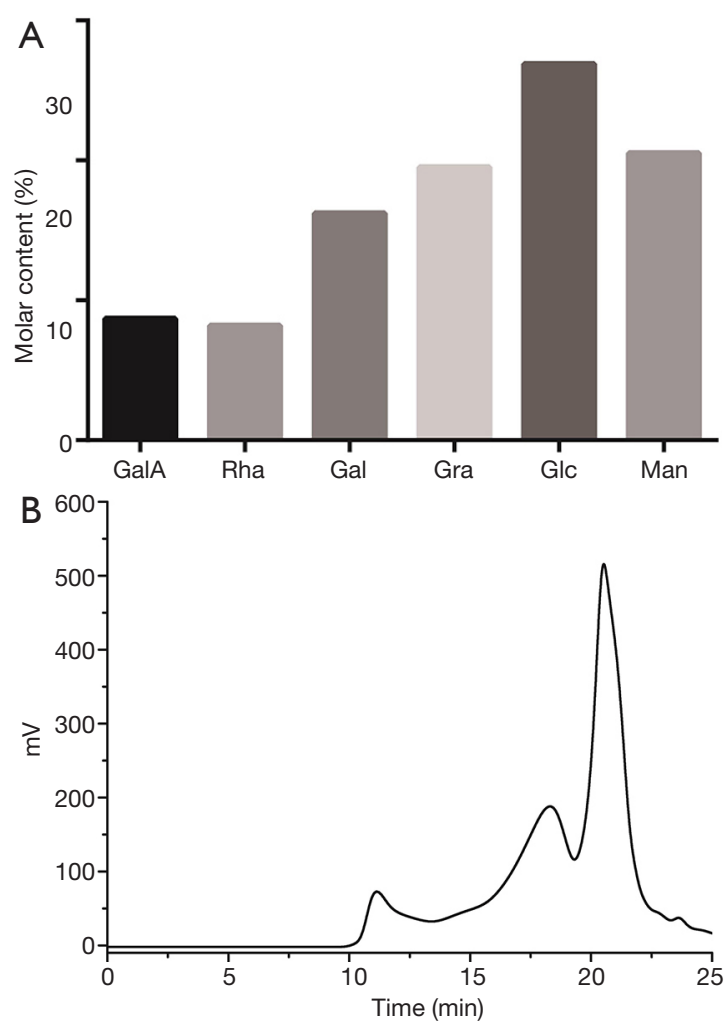

Figure 2 Monosaccharide composition analysis of LJP (A) and the average molecular weight distribution of LJP (B). LJP, Lonicera japonica polysaccharide.

began by placing the mouse in water, using a video camera to record the movement of the mouse. The background color of the device was black. The video analysis system (Noldus Systems, Netherlands) can accurately track the analysis video. When the mouse stops struggling and remains stationary in the water, it is judged to be stationary. It is not recorded within $1 \mathrm{~min}$, record 4 minutes of inactivity time. At the end of the test, dry the mouse hair, and the mice were removed and placed back into the cage. Clean the experimental device and change the water to eliminate the interference of the excrement or odor on the experiment.

\section{Pathological observation}

The mice were deeply anesthetized transcardially perfused with a neutral ( $\mathrm{pH}$ 7.4) phosphate buffer (PBS, 0.1 M) and then replaced with $4 \%$ paraformaldehyde in PBS. After the perfusion was completed, the brain was separated and fixed with $4 \%$ paraformaldehyde for 48 hours at $4{ }^{\circ} \mathrm{C}$. Paraffin-embedded, sliced (4- $\mu \mathrm{m})$, conventional staining of hematoxylin-eosin, or toluidine blue was performed separately. Photographing was conducted under the microscope 100 and 200 times the field of view.

\section{Molecular diagnostic tests-Western blot analysis}

At the end of the experiment, mice that were not perfused were sacrificed by cervical dislocation. Quickly separate the hippocampus in the brain and weigh it. The hippocampus tissue was cut into mini pieces; the RIPA buffer at a concentration of $1 \mathrm{mM} \mathrm{PMSF}$ (presence of protease inhibitor) was added by weight $(10 \mu \mathrm{L} / \mathrm{mg})$ and placed on ice. After standing for 30 minutes, centrifuge at high speed $(12,000 \mathrm{r} / \mathrm{min}, 15 \mathrm{~min})$ at $4{ }^{\circ} \mathrm{C}$. Keep the supernatant for later experiments. The concentration of protein solution was determined at once using the bicinchoninic acid method. An equal amount of protein $(30 \mu \mathrm{g})$ was electrophoretically separated using $10 \%$ to $12 \%$ of SDS-polyacrylamide gels. Then the protein was transferred to a 0.45 pore size PVDF membrane (Millipore) by electrophoresis. The non-specific binding site was blocked with $5 \%$ freshly prepared to skim milk for $1.5 \mathrm{~h}$, the surface of skim milk was washed off, and the membrane was placed in primary anti-working fluid overnight, including rabbit anti-NLRP3 (1:2,000, CST), rabbit antiCaspase1 (1:1,000, CST), rabbit anti-IL-1 $\beta$ (1:1,500, CST), mouse anti- $\beta$-actin (1:5,000, Affinity). Wash off the surface of the primary antibody and place the membrane into the membrane. The corresponding secondary antibody working solution was incubated for $50 \mathrm{~min}$ at room temperature, and the film was chemiluminescent using ECI and exposed to X-ray films. The optical density was quantified by Image J.

\section{Statistical analysis}

The experimental data were expressed as mean \pm standard error mean (SEM). One-way ANOVA and Tukey's HSD test was used to compare the difference of results among the groups. A significance level of $\mathrm{P}<0.05$ was considered statistically significant. SPSS 22.0 software (IBM, Armonk, Chicago, IL, USA) was used to perform statistical analysis.

\section{Results}

\section{Physicochemical property analysis of LFP}

The total carbohydrate, uric acid, and protein contents of LJP were $83.1 \%, 19.3 \%$, and $0.5 \%$, respectively. The 


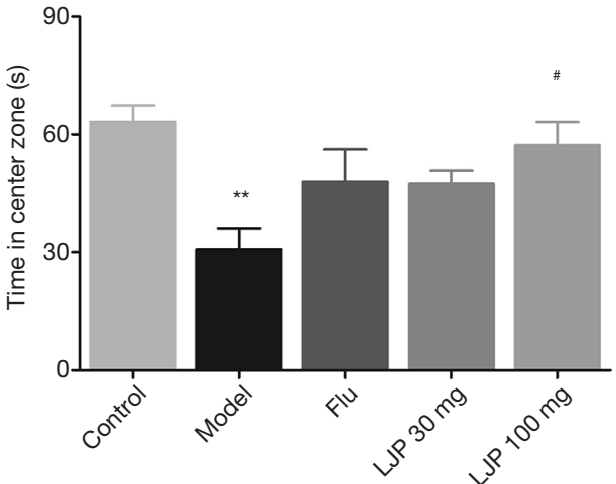

Figure 3 Open field test of LJP. Each point represents the changes compare with respective basal values expressed by percentage $(n=8)$. Significant differences: **, $\mathrm{P}<0.01$ vs. control group; ${ }^{*}, \mathrm{P}<0.05$ vs. model group. LJP, Lonicera japonica polysaccharide.

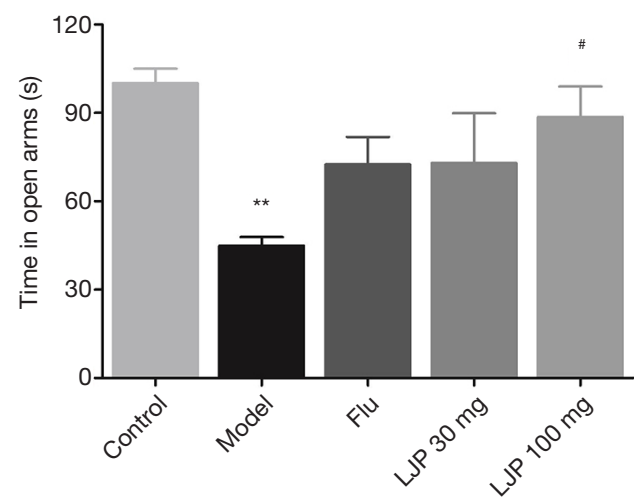

Figure 4 Elevated plus-maze test of LJP. The changes compared with respective basal values expressed by percentage $(n=8)$. Significant differences: ${ }^{* *}, \mathrm{P}<0.01$ vs. control group; ${ }^{*}, \mathrm{P}<0.05$ vs. model group. LJP, Lonicera japonica polysaccharide.

monosaccharide composition of LJP (Figure $2 A$ ) showed that LJP consisted of $8.7 \%$ galacturonic acid (GalA), 8.2\% rhamnose (Rha), $16.2 \%$ galactose $(\mathrm{Gal}), 19.5 \%$ arabinose (Ara), 26.9\% glucose (Glc) and 20.5\% mannose (Man). The ratio of Rha to GalA was 0.94, which fell in the RG-I range of $0.05-1.0$, defined by Schols and Voragen (23), showing that LJP might contain some of RG-I pectin domains. The sum of Gal and Ara's contents was 35.7, showing that LJP might have galactose, arabinose (or arabinogalactose). Besides, LJP also contained a certain amount of Glc and Man, indicating the presence of some glucose and mannose. The molecular weight distribution of LJP (Figure $2 B$ ) suggested that LJP is heterogenous, ranging from 1 to $1,000 \mathrm{kDa}$.

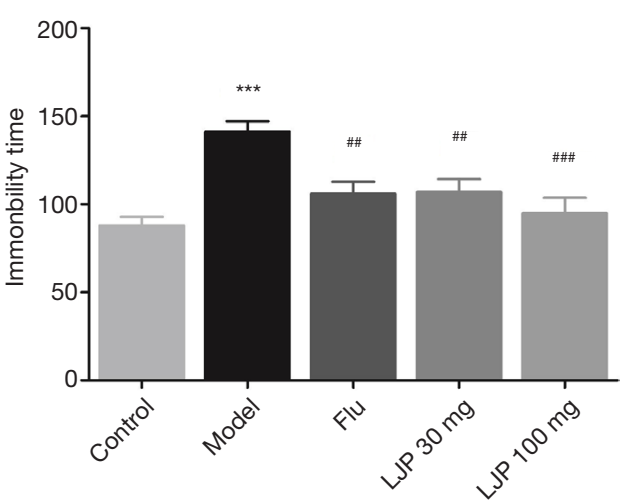

Figure 5 Tail suspension test of LJP. $n=8$. Significant differences: ***, $\mathrm{P}<0.001$ vs. control group; ${ }^{\#}, \mathrm{P}<0.01,{ }^{\# \#}, \mathrm{P}<0.001$ vs. model group. LJP, Lonicera japonica polysaccharide.

\section{Behavioral tests of LFP in protecting depressed mice}

\section{Model establishment}

In the open field test, the mice of the Model group showed a significant decrease in time of the mouse in the center zone as compared to those in the Control group. In the test of the elevated plus-maze, the time taken of mice stayed in the open arm decreased significantly. However, the immobility time increased significantly in tail suspension and forced arm tests. These results indicated that the chronic unpredictable, stimulating depression mice model was successfully established.

\section{Open field test}

Based on Figure 3, the time of the mice model in the center zone was significantly reduced when compared with the Control group $(\mathrm{P}<0.01)$. The mice from the positive drug group (Flu group) and $30 \mathrm{mg}$ LJP group showed an improvement trend by increasing the time of mice in the center zone, but the change was not significant. In contrast, a high dose of LJP significantly increased the time of depressed mice in the center zone $(\mathrm{P}<0.05)$.

\section{Elevated plus maze test}

As shown in Figure 4, the depressed mice had significantly reduced the time in the open arms as compared to those in the Control group $(\mathrm{P}<0.01)$. All three drug treatment groups increased the time of mice in the open arms. A high dose of LJP significantly increased the time of depressed mice in the center zone $(\mathrm{P}<0.05)$.

\section{Tail suspension test}

Based on Figure 5, the immobility time of the mice with 
Page 6 of 9

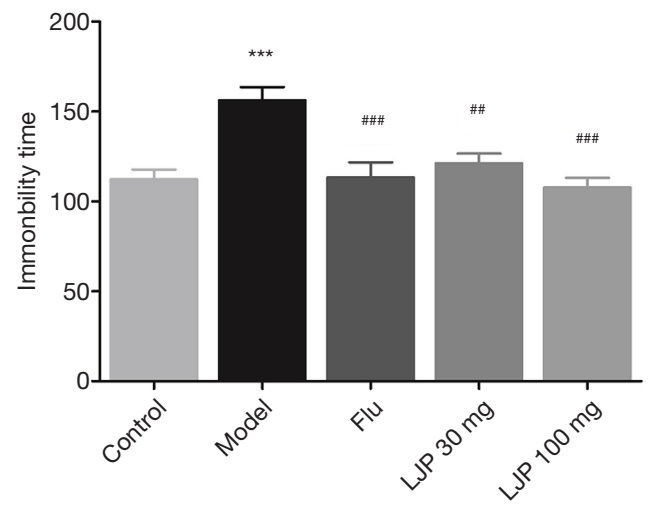

Liu et al. LJP inhibited mice depression by regulating NLRP3 inflammasome

depression was significantly prolonged compared with the healthy mice $(\mathrm{P}<0.001)$. In contrast, the Flu group $(\mathrm{P}<0.01)$ and LJP groups dramatically reduced the immobility time of the depressed mice $(\mathrm{P}<0.01, \mathrm{P}<0.001)$.

\section{Forced swim test}

As shown in Figure 6, the immobility time of the mice with depression was significantly prolonged compared with the healthy mice $(\mathrm{P}<0.001)$. On the other hand, the drug treatment groups significantly decreased the immobility time of the depressed mice (Flu, $\mathrm{P}<0.001$; LJP, $\mathrm{P}<0.01, \mathrm{P}<0.001)$.

\section{Pathological examination of LFP protection on depressed mice}

As shown in Figure 7, compared with the control group,
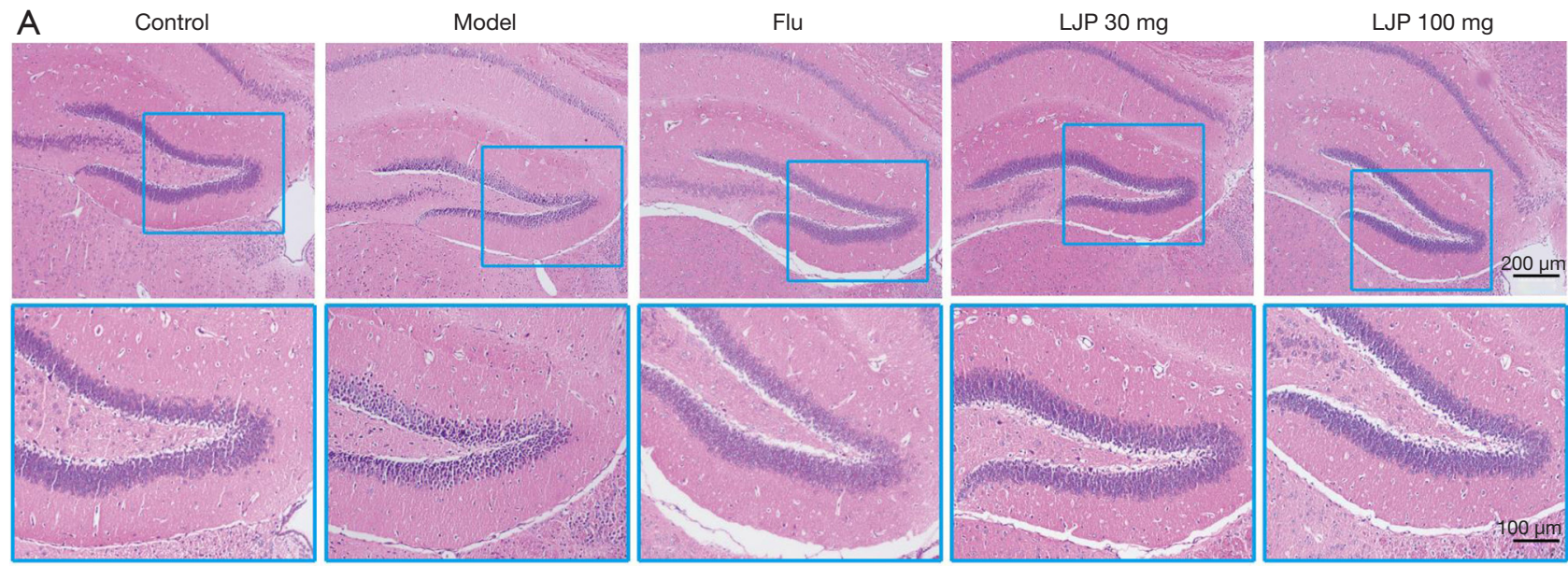

B
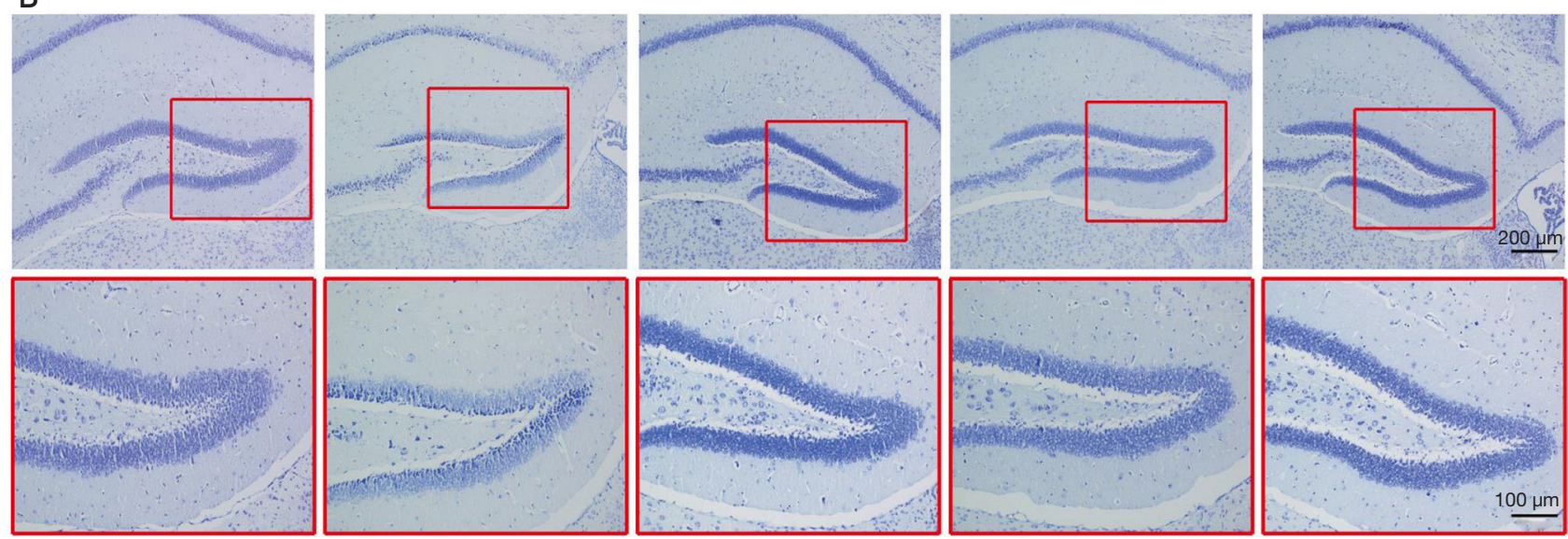

Figure 7 Pathological examination of the LJP protection effect. (A) HE staining; (B) toluidine blue staining. LJP, Lonicera japonica polysaccharide. 

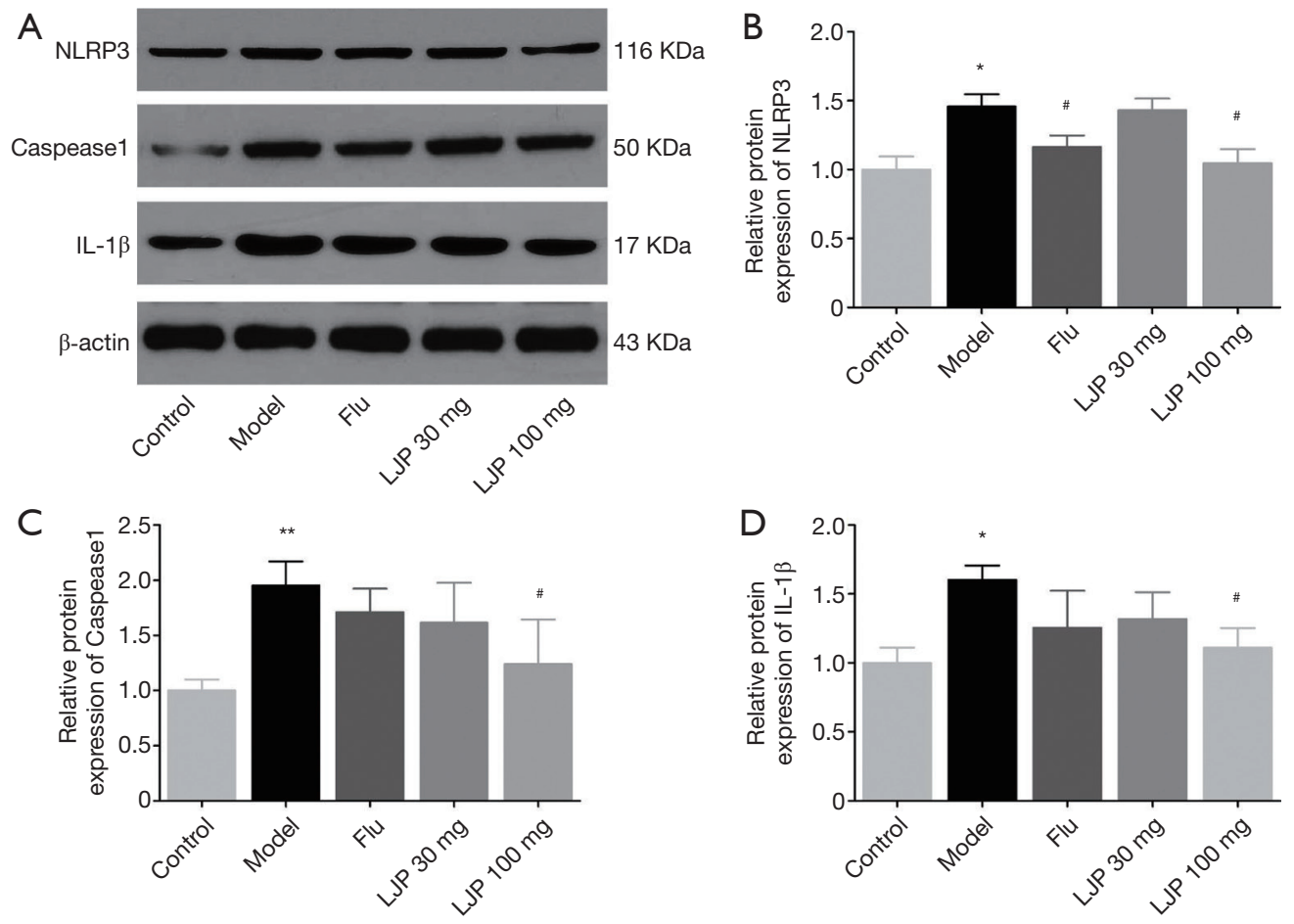

Figure 8 Western blot analysis of the LJP protection effect. The changes compare with respective basal values expressed by percentage ( $\mathrm{n}=3$ ). Significant differences: *, $\mathrm{P}<0.05$, ** $\mathrm{P}<0.01$ vs. control group; ${ }^{*}, \mathrm{P}<0.05$ vs. model group. LJP, Lonicera japonica polysaccharide.

the model group found that the number of nerve cells decreased, the nerve level was unclear, and the arrangement lacked regularity; the nerve cells were deformed, necrosis occurred, and the cell structure was not clear. The positive group (Flu group) and LJP groups significantly improved the pathology results.

\section{Western blot analysis of LFP protection on depressed mice}

As shown in Figure 8, The protein expressions of NLRP3, caspase- 1 , and IL- $1 \beta$ in mice's hippocampus of depressed mice were significantly upregulated as compared to those in the Control group (NLRP3, $\mathrm{P}<0.05$; Caspease1, $\mathrm{P}<0.01$; IL-1 $\beta, \mathrm{P}<0.05)$. The Flu group and LJP groups significantly inhibited such upregulation. However, the effect of Flu and LJP was not synchronous. The Flu group and LJP groups significantly reduce the expressions of NLRP3 $(\mathrm{P}<0.05)$; the LJP $100 \mathrm{mg} / \mathrm{kg}$ group significantly reduce the expressions of Caspease 1 and IL-1 $\beta(\mathrm{P}<0.05)$. The effect of LJP was more significant, suggesting that this pathway might not be the main target of positive drug action, but the effect of LJP was closely related to this pathway.

\section{Discussion}

Work and love are two areas of people's lives from which we find meaning and get satisfaction. However, they can also become our life-threatening stressors due to significant aspects such as rapid economic change (24). Work pressure and life pressure are increasing with social development, which delivers increasing stress and depression. The pathogenesis of depression is complex and related to emotions. Everything can cause mood disorder, mental stress, trauma, and unpleasant experience, which results in depression. The chronic unpredictable mild stress (CUMS) mice model has similar behavioral and physiological aspects that are relevance to clinically human depression (25). Although depression is a mental illness, the expression of inflammatory bodies based on Western blot analysis indicates that depression has led to physical damage to the human body rather than being limited to emotion disorder.

The occurrence of depression may be due to the presence of NLRP3 inflammasome, which is related to systemic immunity and is a vital component of innate immunity. It was found that the activation of the inflammatory system in the CNS of patients with severe depression, accompanied 
by abnormally elevated levels of inflammatory cytokines (such as IL-1 $\beta$ and TNF- $\alpha$ ), and in the study of peripheral blood in patients with depression, mononuclear The degree of NLRP3 activation in cells is significantly higher than in healthy people (26). According to the previous study, significant changes in NLRP3 were found in both the peripheral and central nervous systems of depressed mice (27). In contrast, the CUMS mice treated with LJP had significant differences in behavioral tests and Western blot analysis as compared with untreated mice, speculating that the occurrence of depression is related to NLRP3 inflammasomes.

The previous study had shown that the autophagy response of cells has a regulatory effect on the activation of inflammatory bodies. The main manifestation is that inflammatory bodies can induce autophagy, and autophagy has a negative regulatory effect on inflammatory bodies (28). It is speculated that LJP may inhibit the occurrence of depression by regulating the NLRP3 inflammatory pathway.

\section{Conclusions}

LJP exhibited a strong protective effect on mice with depression, via inhibiting NLRP3 inflammasome pathway. The results will help to understand the potential use of Lonicera japonica Tbun. polysaccharide in pharmaceutical and food applications better.

\section{Acknowledgments}

Funding: National Natural Science Foundation of Chinafunded this research (No. 31760251), the Technology Research and Development Program of Guizhou [No. qiankehezhicheng (2018)2803], the Foundation for Young Scientists Growth Project of Guizhou Province (No. qianjiaoheKYzi2017192) (No. qianjiaoheKYzi2017199), the Program for Excellent Young Talents of Zunyi Medical University (No. 18zy-006), and the Doctoral Scientific Research Starting Foundation of Affiliated Hospital of Zunyi Medical University (No. yuanzi201607).

\section{Footnote}

Conflicts of Interest: The authors have no conflicts of interest to declare.

Ethical Statement: The authors are accountable for all aspects of the work in ensuring that questions related to the accuracy or integrity of any part of the work are appropriately investigated and resolved. The experimental protocol was approved and approved by the Ethics Committee of Zunyi Medical University.

\section{References}

1. Brigitta B. Pathophysiology of depression and mechanisms of treatment. Dialogues Clin Neurosci 2002;4:7-20.

2. Wang Y, Song B, Zhang Y, et al. Evaluation and predictive factors analyses for patient-self-reported depression, anxiety and quality of life in patients with metastatic renal cell carcinoma underwent interferon- $\alpha$ treatment: a prospective cohort study. Transl Cancer Res 2018;7:1460-73.

3. Dallé E, Mabandla MV. Early Life Stress, Depression And Parkinson's Disease: A New Approach. Mol Brain 2018;11:18.

4. Jeon SA, Lee E, Hwang I, et al. NLRP3 Inflammasome Contributes to Lipopolysaccharide-induced DepressiveLike Behaviors via Indoleamine 2,3-dioxygenase Induction. Int J Neuropsychopharmacol 2017;20:896-906.

5. Song B, Zhang Y, Wang Y, et al. Intensive patients' care program ameliorates anxiety and depression, and sustained anxiety correlates with worse overall survival in renal cell carcinoma patients underwent radical nephrectomy. Transl Cancer Res 2018;7:1601-13.

6. Gardner A,Johansson A, Wibom R, Nennesmo I, von Döbeln U, Hagenfeldt L, Hällström T. Alterations of mitochondrial function and correlations with personality traits in selected major depressive disorder patients. J Affect Disorders 2003;76:55-68.

7. Maes M, Bosmans E, Meltzer HY, et al. Interleukin-1 beta: a putative mediator of HPA axis hyperactivity in major depression? Am J Psychiat 1993;150:1189-93.

8. Maes M. Evidence for an immune response in major depression: a review and hypothesis. Prog Neuropsychopharmacol Biol Psychiatry 1995;19:11-38.

9. Rawdin BJ, Mellon SH, Dhabhar FS, et al. Dysregulated relationship of inflammation and oxidative stress in major depression. Brain Behav Immun 2013;31:143-52.

10. Dowlati Y, Herrmann N, Swardfager W, et al. A metaanalysis of cytokines in major depression. Biol Psychiat 2010;67:446-57.

11. Abbasi SH, Hosseini F, Modabbernia A, et al. Effect of celecoxib add-on treatment on symptoms and serum IL-6 concentrations in patients with major depressive disorder: randomized double-blind placebo-controlled study. J 
Affect Disorders 2012;141:308-14.

12. Hannestad J, Della Gioia N, Bloch M. The effect of antidepressant medication treatment on serum levels of inflammatory cytokines: a meta-analysis. Neuropsychopharmacology 2011;36:2452-9.

13. Müller N, Schwarz M, Dehning S, et al. The cyclooxygenase-2 inhibitor celecoxib has therapeutic effects in major depression: results of a double-blind, randomized, placebo controlled, add-on pilot study to reboxetine. Mol Psychiatry 2006;11:680-4.

14. Nery FG, Monkul ES, Hatch JP, et al. Celecoxib as an adjunct in the treatment of depressive or mixed episodes of bipolar disorder: a double-blind, randomized, placebocontrolled study. Hum Psychopharmacol 2008;23:87-94.

15. Schroder K, Tschopp J. The inflammasomes. Cell 2010;140:821-32.

16. Wang C, Wang G, Liu H, et al. Protective effect of bioactive compounds from Lonicera japonica Thunb. against $\mathrm{H} 2 \mathrm{O} 2$-induced cytotoxicity using neonatal rat cardiomyocytes. Iran J Basic Med Sci 2016;19:97-105.

17. Park HS, Park KI, Lee DH, et al. Polyphenolic extract isolated from Korean Lonicera japonica Thunb. induce G2/M cell cycle arrest and apoptosis in HepG2 cells: involvements of PI3K/Akt and MAPKs. Food Chem Toxicol 2012;50:2407-16.

18. Shang X, Pan H, Li, M, et al. Lonicera japonica Thunb.: ethnopharmacology, phytochemistry and pharmacology of an important traditional Chinese medicine. J. Ethnopharmacol 2011;138:1-21.

19. Tzeng TF, Liou SS, Chang CJ, et al. The ethanol extract of Lonicera japonica (Japanese honeysuckle) attenuates diabetic nephropathy by inhibiting p-38 MAPK activity

Cite this article as: Liu P, Bai X, Zhang T, Zhou L, Li J, Zhang L. The protective effect of Lonicera japonica polysaccharide on mice with depression by inhibiting NLRP3 inflammasome. Ann Transl Med 2019;7(24):811. doi: 10.21037/ atm.2019.12.64 in streptozotocin-induced diabetic rats. Planta Med 2014;80:121-9.

20. Weon JB, Yang HJ, Lee B, et al. Neuroprotective activity of the methanolic extract of Lonicera japonica in glutamate-injured primary rat cortical cells. Pharmacogn Mag 2011;7:284-8.

21. Xiong J, Li S, Wang W, et al. Screening and identification of the antibacterial bioactive compounds from Lonicera japonica Thunb. leaves. Food Chem 2013;138:327-33.

22. Zhang T, Xiang J, Zheng G, et al. Preliminary characterization and anti-hyperglycemic activity of a pectic polysaccharide from okra (Abelmoschus esculentus (L.) Moench). J Funct Foods 2018;41:19-24.

23. Schols H, Voragen A. Complex pectins: structure elucidation using enzymes. Prog Biotechnol 1996;14:3-19.

24. Woo JM, Postolache TT. The impact of work environment on mood disorders and suicide: Evidence and implications. Int J Disabil Hum Dev 2008;7:185-200.

25. Willner P. The chronic mild stress (CMS) model of depression: history, evaluation and usage. Neurobiol Stress 2016;6:78-93.

26. Alcocer-Gómez E, de Miguel M, Casas-Barquero N, et al. NLRP3 inflammasome is activated in mononuclear blood cells from patients with major depressive disorder. Brain Behav Immun 2014;36:111-7.

27. Su WJ, Zhang Y, Chen Y, et al. NLRP3 gene knockout blocks NF- $\kappa \mathrm{B}$ and MAPK signaling pathway in CUMSinduced depression mouse model. Behav Brain Res 2017;322:1-8.

28. Motta V, Soares F, Sun T, et al. NOD-like receptors: versatile cytosolic sentinels. Physiol Rev 2015;95:149-78. 